\title{
Contamination par Salmonella SPP. Des Plats PRÉPARÉs À base DE PORC DANS LES GARGOTES D'ANTANANARIVO (MADAGASCAR) ET DÉTERMINATION DES FACTEURS DE RISQUE ASSOCIÉS *
}

\author{
SALMONELLA CONTAMINATION OF PORK MEAT DISHES FROM STREET RESTAURANTS IN \\ ANTANANARIVO (MADAGASCAR) AND DETERMINATION OF ASSOCIATED RISK FACTORS
}

\author{
Contaminación por Salmonella de platos preparados a base de CARNe de Cerdo \\ EN RESTAURANTES DE CALLE EN ANTANANARIVO (MADAGASCAR) \\ Y DETERMINACIÓN DE LOS FACTORES DE RIESGO ASOCIADOS
}

\author{
C. Abat ${ }^{1}$ M. Rakotoharinome ${ }^{2}$ M. Maeder ${ }^{3}$ B. Contamin ${ }^{3}$ V. Porphyre ${ }^{4}$ E. Cardinale ${ }^{1,5 *}$
}

Mots-clés : Viande porcine - Salmonella - Facteur de risque Contamination - Madagascar.

Keywords: Pork - Salmonella - Risk factor - Contamination Madagascar.

Palabras clave: Carne de cerdo - Salmonella - Factor de riesgo - Contaminación - Madagascar.

es toxi-infections alimentaires sont un problème majeur pour Ltous les Etats de la planète. Parmi les causes principales se trouvent Salmonella et Campylobacter, présents dans la nourriture vendue dans les restaurants de rue. Madagascar, plus grande île de l'océan Indien, possède peu de données exploitables pour quantifier l'ampleur de cette contamination. Or, ce pays possède une importante population porcine dont une partie de la viande est servie dans les plats des gargotes en ville. Pour ces raisons, le gouvernement malgache souhaite une meilleure maîtrise de la qualité microbiologique des produits. Cette étude a été conçue dans cette optique et regroupe la direction des services vétérinaires de Madagascar, le Cirad, la faculté de médecine et le Centre d'infectiologie Charles Mérieux d'Antananarivo.

Les objectifs étaient de déterminer la prévalence potentielle de Salmonella spp. et de Campylobacter spp. directement présents dans les plats cuisinés à base de porc, vendus par les restaurants de rue de la capitale, ainsi que de caractériser les facteurs de risque à l'origine des contaminations des plats par ces bactéries.

1. Cirad, UMR Cmaee, 2 rue Maxime Rivière, 97491 Sainte-Clotilde, Réunion, France.

2. Services vétérinaires de Madagascar, ministère de l'Elevage,

rue Farafaty Ampandrianomby, Antananarivo, Madagascar.

3. Fondation Mérieux, Antananarivo, Madagascar.

4. Cirad, UMR Selmet, station de Ligne-Paradis, 7 chemin de l'IRAT, 97410 Saint-Pierre, Réunion, France.

5. Centre de recherche et de veille sur les maladies émergentes dans l'océan Indien, Cyroi, 97491 Sainte-Clotilde Cedex, Réunion, France.

* Auteur pour la correspondance

E-mail : eric.cardinale@cirad.fr

ॠ Ce texte est issu d'une communication orale présentée aux journées scientifiques QualiREG 2012. L'ensemble des résultats a ensuite fait l'objet d'une publication scientifique (Cardinale et coll., 2015).
Pour la réaliser, soixante gargotes sélectionnées aléatoirement dans treize quartiers d'Antananarivo ont été visitées sur une période de trois mois. Les propriétaires des établissements ont été questionnés sur leurs pratiques de préparation et trois plats à base de porc ont été achetés à chacun d'eux. Chaque échantillon récolté a ensuite été traité suivant un protocole comprenant un pré-enrichissement non sélectif suivi d'un pré-enrichissement en bouillons sélectifs. Les colonies suspectées d'être du genre Salmonella ou Campylobacter ont été récupérées à partir de géloses sélectives, confirmées ou infirmées par tests biochimiques, puis par la méthode RapID ONE, et enfin isolées. Salmonella a ensuite été sérotypée selon la méthode de Kaufmann White Le Minor (2). En cas de contamination confirmée d'un plat échantillonné, le restaurant dont il était issu était défini comme contaminé, constituant ainsi la variable à expliquer. Dès lors, une analyse statistique en plusieurs étapes était réalisée afin de déterminer quelles pratiques de préparation des établissements enquêtés devaient être considérées comme des facteurs de risque.

La confirmation des bactéries suspectes par la méthode RapID ONE a permis d'identifier uniquement neuf plats contaminés par des salmonelles. Aucun Campylobacter n'a été détecté. Les sérotypages réalisés ont mis en évidence trois sérotypes : Salmonella Typhimurium, Salmonella Newport et Salmonella Seftenberg. L'analyse globale des pratiques des gargotiers enquêtés a montré que ces derniers présentaient certaines lacunes concernant les bonnes pratiques d'hygiène à appliquer dans leur secteur d'activité. Enfin, l'analyse des facteurs de risque a révélé que, dans le contexte de l'étude, I'utilisation des nappes (odds ratio $[\mathrm{OR}]=8,83$; intervalle de confiance [IC] $95 \%:[1,24-62,2])$ et la température de service des plats $(\mathrm{OR}=5,41$; IC $95 \%$ : $[1,25-35,22])$ étaient fortement associées à l'augmentation du risque de contamination des plats par Salmonella. A I'inverse, la situation géographique du quartier de l'établissement enquêté (OR = 0,15; IC $95 \%$ : [0,022-0,98]), le type de construction (OR $=0,03 ;$ IC $95 \%:[0,0025-0,29])$, le port d'habits spécifiques $(\mathrm{OR}=0,15 ; \mathrm{IC} 95 \%$ : [0,018-0,99]) et de couvre-chef intégral par le personnel $(\mathrm{OR}=0,05$; IC $95 \%$ : [0,004-0,57]) ont semblé associés à une diminution des risques de contamination des plats par les salmonelles. Campylobacter n'a pas été retrouvé en raison notamment de sa sensibilité à la chaleur ; il a été vraisemblablement détruit par la cuisson. Une étape de sensibilisation a été engagée pour améliorer les pratiques et les propriétaires des restaurants ont été très réceptifs aux conseils prodigués. 


\section{Tableau I}

Modèle final de régression logistique pour les facteurs de risque de contamination par Salmonella dans les restaurants de rue $(n=60)$ à Antananarivo, Madagascar, en 2012

\begin{tabular}{|c|c|c|c|c|}
\hline \multirow[t]{2}{*}{$\begin{array}{l}\text { Variables conservées } \\
\text { dans le modèle final }\end{array}$} & \multirow[t]{2}{*}{ Valeur $\mathbf{p}$} & \multirow[t]{2}{*}{$\begin{array}{l}\text { Restaurants positifs } \\
\text { pour Salmonella a }(\%)\end{array}$} & \multicolumn{2}{|c|}{$\begin{array}{c}\text { Modèle de régression } \\
\text { logistique }\end{array}$} \\
\hline & & & Odds ratio & IC b à 95 \% \\
\hline Locaux spécifiques pour le restaurant & 0,08 & & & \\
\hline Non & & 33,4 & 1 & - \\
\hline Oui & & 5,8 & 0,03 & $0,0025-0,29$ \\
\hline Vêtements spécifiques pour le personnel & 0,22 & & & \\
\hline Non & & 17,1 & 1 & - \\
\hline Oui & & 0 & 0,05 & $0,004-0,57$ \\
\hline Utilisation de nappes & 0,13 & & & \\
\hline Non & & 6,5 & 1 & - \\
\hline Oui & & 21,4 & 8,83 & $1,24-62,2$ \\
\hline Température observée dans les plats à base de porc & 0,21 & & & \\
\hline$<52,5^{\circ} \mathrm{C}$ incluse & & 12,0 & 5,41 & $1,25-35,22$ \\
\hline$>52,5^{\circ} \mathrm{C}$ & & 0 & 1 & - \\
\hline
\end{tabular}

${ }^{\text {a }}$ Basé sur le nombre total de restaurants dans la classe restaurants contaminés

${ }^{\mathrm{b}}$ Intervalle de confiance

\section{BIBLIOGRAPHIE}

1. CARDINALE E., ABAT C., CONTAMIN B., PORPHYRE V., RAKOTOHARINOME M., MAEDER M., 2015. Salmonella and Campylobacter contamination of ready-to-eat street-vended pork meat dishes in Antananarivo, Madagascar: a risk for the consumers? Foodborne Pathog. Dis., 12: 197-202.
2. GRIMONT P.A.D., WEILL F.-X., 2007. Antigenic formulae of the Salmonella serovars, 9th Edn, World Health Organization Collaborating Centre for Reference and Research on Salmonella. Paris, France, Institut Pasteur.

Accepted 30 April 2015; Online publication June 2015 